\title{
Sharp lower bounds involving circuit layout system
}

\author{
Jiajin Wen ${ }^{1}$, Shanhe $\mathrm{Wu}^{2 *}$ and Chaobang Gao
}

${ }^{*}$ Correspondence:

shanhewu@gmail.com

${ }^{2}$ Department of Mathematics and Computer Science, Longyan

University, Longyan, Fujian 364012 , China

Full list of author information is available at the end of the article

\begin{abstract}
The circuit layout system $\operatorname{CLS}\left\{\Gamma_{n}(\mathbf{A}), \Gamma_{N}\left(\mathbf{A}^{*}\right), \delta\right\}_{\mathbb{E}}$ in a Euclidean space $\mathbb{E}$ is defined. By means of algebraic, analytic, geometric and inequality theories, we obtain several sharp lower bounds involving the circuit layout system.
\end{abstract}

MSC: $51 \mathrm{~K} 05 ; 26 \mathrm{D} 15$

Keywords: circuit layout system; Euclidean space; Minkowski's inequality

\section{Introduction}

We first introduce a circuit layout problem as follows. Let $\Gamma$ be a rectangular (or polygon) courtyard (or street). Five light poles (or street lamp), with a fixed minimal distance apart from each other, are proposed to be erected on the boundary of $\Gamma$, and straight underground pipes are planned to connect these poles (see Figure 1). Assuming that the major cost of the construction project is the price of the pipes, it is then important to find out the minimal total lengths required for the project, its purpose is to estimate the installation costs.

We can easily illustrate this problem by means of Figure 1 in a later Example 4.3 in which the corners of the courtyard $\Gamma$ are indicated by the points $A_{1}, A_{2}, A_{3}$ and $A_{4}$, while the light poles are indicated by $A_{1}^{*}, A_{2}^{*}, A_{3}^{*}, A_{4}^{*}$ and $A_{5}^{*}$, respectively. The light poles are kept apart from each other for clear reasons so that we may assume the distances

$$
\left\|A_{2}^{*}-A_{1}^{*}\right\|,\left\|A_{3}^{*}-A_{2}^{*}\right\|,\left\|A_{4}^{*}-A_{3}^{*}\right\|,\left\|A_{5}^{*}-A_{4}^{*}\right\|,\left\|A_{5}^{*}-A_{1}^{*}\right\| \geq \delta>0 .
$$

We need to find among all possible locations of $A_{1}^{*}, \ldots, A_{5}^{*}$ such that the total length

$$
\left\|A_{2}^{*}-A_{1}^{*}\right\|+\left\|A_{3}^{*}-A_{2}^{*}\right\|+\left\|A_{4}^{*}-A_{3}^{*}\right\|+\left\|A_{5}^{*}-A_{4}^{*}\right\|+\left\|A_{5}^{*}-A_{1}^{*}\right\|
$$

is the minimal one.

The above problem can easily be generalized. To this end, we need to recall some basic concepts as follows.

Let $\mathbb{E}$ be a Euclidean space, and $\alpha, \beta \in \mathbb{E}$. The inner product of $\alpha$ and $\beta$ is denoted by $\langle\alpha, \beta\rangle$ and the norm of $\alpha$ is denoted by $\|\alpha\|$. The dimension $\operatorname{dim} \mathbb{E}$ of $\mathbb{E}$ satisfies $\operatorname{dim} \mathbb{E} \geq n$ if and only if there exist $n$ linearly independent vectors $\boldsymbol{\varepsilon}_{1}, \boldsymbol{\varepsilon}_{2}, \ldots, \boldsymbol{\varepsilon}_{n}$ in $\mathbb{E}$ (see [1]).

Let $B, C$ be points in $\mathbb{E}$, the closed, open and closed-open segments joining them will respectively be denoted by

$$
[B C], \quad(B C), \quad[B C) \text { and } \quad(B C]
$$

○2013 Wen et al.; licensee Springer. This is an Open Access article distributed under the terms of the Creative Commons Attribution License (http://creativecommons.org/licenses/by/2.0), which permits unrestricted use, distribution, and reproduction in any medium, provided the original work is properly cited. 
Figure 1 The graph of $\operatorname{CLS}\left\{\Gamma_{4}(A), \Gamma_{5}\left(A^{*}\right), 2\right\}_{\mathbb{R}^{2}}$.

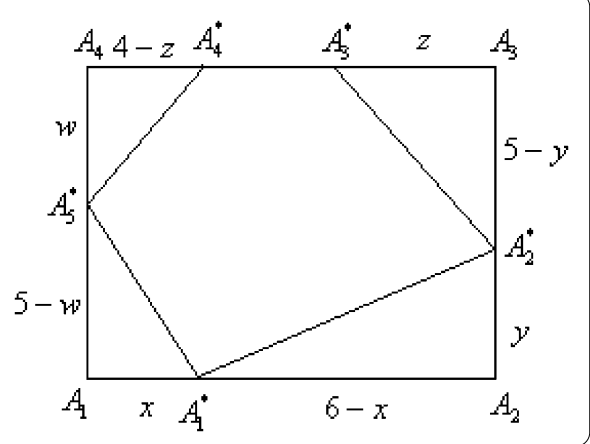

and defined as usual by

$$
\begin{aligned}
& \left\{\chi_{B, C}(t) \mid t \in[0,1]\right\}, \quad\left\{\chi_{B, C}(t) \mid t \in(0,1)\right\}, \\
& \left\{\chi_{B, C}(t) \mid t \in[0,1)\right\} \quad \text { and } \quad\left\{\chi_{B, C}(t) \mid t \in(0,1]\right\},
\end{aligned}
$$

where

$$
\chi_{B, C}(t):=(1-t) B+t C
$$

Let $\operatorname{dim} \mathbb{E} \geq 2, \mathbf{A}=\left(A_{1}, A_{2}, \ldots, A_{n}\right) \in \mathbb{E}^{n}$, where

$$
A_{i} \neq A_{i+1}, \quad i=1,2, \ldots, n, n \geq 3,
$$

be a sequence of points in $\mathbb{E}$ and

$$
A_{i}=A_{j} \quad \Leftrightarrow \quad i \equiv j(\bmod n), \quad i, j=0, \pm 1, \pm 2, \ldots
$$

We call the set

$$
\Gamma_{n}(\mathbf{A})=\bigcup_{i=1}^{n}\left[A_{i} A_{i+1}\right)
$$

an $n$-polygon, or a polygon if no confusion is caused. The angle ${ }^{\mathrm{a}}$ at $A_{i}$ and the angle $\angle A$ are defined as

$$
\angle A_{i}:=\angle\left(A_{i}-A_{i-1}, A_{i+1}-A_{i}\right), \quad i=1,2, \ldots, n \quad \text { and } \quad \angle A:=\min _{1 \leq i \leq n}\left\{\angle A_{i}\right\}
$$

In case each $\angle A_{i}$ is the same, we say that our polygon is equiangular. We will also denote the total length (or perimeter) of an $n$-gon by

$$
\left|\Gamma_{n}(\mathbf{A})\right|=\sum_{i=1}^{n} a_{i}=\sum_{i=1}^{n}\left\|A_{i+1}-A_{i}\right\|,
$$

where, and in the future,

$$
a_{i}:=\left\|A_{i+1}-A_{i}\right\|, \quad i=1,2, \ldots, n .
$$


Now we give the definition of the circuit layout system in a Euclidean space as follows.

Definition 1.1 Let $\Gamma_{n}(\mathbf{A})$ and $\Gamma_{N}\left(\mathbf{A}^{*}\right)$, where $N \geq n \geq 3$, be two polygons in $\mathbb{E}$ with the dimension $\operatorname{dim} \mathbb{E} \geq 2$. We say that the set

$$
\operatorname{CLS}\left\{\Gamma_{n}(\mathbf{A}), \Gamma_{N}\left(\mathbf{A}^{*}\right), \delta\right\}_{\mathbb{E}}:=\left\{\Gamma_{n}(\mathbf{A}), \Gamma_{N}\left(\mathbf{A}^{*}\right), \delta\right\}
$$

is a circuit layout system (or CLS for short) if the following conditions are satisfied:

(H1.1) $\angle A_{i} \in(0, \pi), i=1,2, \ldots, n$.

(H1.2) $A_{j}^{*} \in \Gamma_{n}(\mathbf{A})$ for $j \in\{1,2, \ldots, N\}$ and $A_{1}^{*} \in\left[A_{1} A_{2}\right)$.

(H1.3) If $A_{j}^{*}, A_{j+1}^{*} \in\left[A_{i} A_{i+1}\right)$, then $A_{j+1}^{*} \in\left(A_{j}^{*} A_{i+1}\right)$ for $i=1,2, \ldots, n$ and $j=1,2, \ldots, N$.

(H1.4) If $A_{j}^{*} \in\left[A_{i} A_{i+1}\right)$ and $A_{k}^{*} \in\left[A_{i+1} A_{i+2}\right)$ for $j, k \in\{1,2, \ldots, N\}$ and $i \in\{1,2, \ldots, n\}$, then $j<k$.

(H1.5) For any $i \in\{1,2, \ldots, n\}$, there exists $j \in\{1,2, \ldots, N\}$ such that $A_{j}^{*} \in\left[A_{i} A_{i+1}\right)$.

(H1.6) For any $j \in\{1,2, \ldots, N\}$, there is $\delta>0$ such that

$$
\left\|A_{j+1}^{*}-A_{j}^{*}\right\| \geq \delta .
$$

In this paper, we are concerned with the sharp lower bound (see [2-5]) of $\left|\Gamma_{N}\left(\mathbf{A}^{*}\right)\right|$, its purpose is to estimate the installation costs of the circuit layout problem. In other words, we will mainly be concerned with the following problem.

Problem 1.1 (Circuit layout problem) Let $\operatorname{CLS}\left\{\Gamma_{n}(\mathbf{A}), \Gamma_{N}\left(\mathbf{A}^{*}\right), \delta\right\}_{\mathbb{E}}$ be a CLS. How can we determine the lower bound of $\left|\Gamma_{N}\left(\mathbf{A}^{*}\right)\right|$ by means of $n, N, \delta$ and $\Gamma_{n}(\mathbf{A})$ ?

In this paper, by means of algebraic, analytic, geometric and inequality theories, several sharp lower bounds of $\left|\Gamma_{N}\left(\mathbf{A}^{*}\right)\right|$ in Problem 1.1 are obtained. As applications of our results, in Section 4, we calculate that $\inf \left\{\left|\Gamma_{N}\left(\mathbf{A}^{*}\right)\right|\right\}$ for the special circuit layout system $\operatorname{CLS}\left\{\Gamma_{n}(\mathbf{A}), \Gamma_{N}\left(\mathbf{A}^{*}\right), \delta\right\}_{\mathbb{R}^{2}}$ by means of three effective examples.

\section{Preliminaries}

We provide in this section some basic terminologies and results which are necessary for the investigation of Problem 1.1.

We first recall the concept of parallel vectors for later use. Two vectors $\mathbf{x}$ and $\mathbf{y}$ in $\mathbb{E}$ are said to be in the same (opposite) direction if (i) $\mathbf{x}=0$ or $\mathbf{y}=0$, or (ii) $\mathbf{x} \neq 0$ and $\mathbf{y} \neq 0$ and $\mathbf{x}$ is a positive (respectively negative) constant multiple of $\mathbf{y}$. Two vectors $\mathbf{x}$ and $\mathbf{y}$ in the same (opposite) direction are indicated by $\mathbf{x} \uparrow \mathbf{y}$ (respectively $\mathbf{x} \downarrow \mathbf{y}$ ) (see [1]).

Next, we set that

$$
\mathbf{x}=\left(x_{1}, x_{2}, \ldots, x_{n}\right)^{T}, \quad\|\mathbf{x}\|_{p}=\left(\left|x_{1}\right|^{p}+\left|x_{2}\right|^{p}+\cdots+\left|x_{n}\right|^{p}\right)^{1 / p},
$$

where $p \in(1, \infty)$.

In order to study Problem 1.1, we need six lemmas as follows.

Lemma 2.1 (Minkowski's inequality [6]) If $\mathbf{x}, \mathbf{y} \in \mathbb{R}^{n}$ and $p \in(1, \infty)$, then

$$
\|\mathbf{x}+\mathbf{y}\|_{p} \leq\|\mathbf{x}\|_{p}+\|\mathbf{y}\|_{p} .
$$

Furthermore, the equality holds if and only if $\mathbf{x} \uparrow \mathbf{y}$. 
According to Lemma 2.1 and the algebraic theory, we easily get the following lemma.

Lemma 2.2 (Minkowski-type inequality [6]) Let $A \in \mathbb{R}^{n \times n}$. If $A^{T}=A, A \geq 0$ and $f(\mathbf{x})=$ $\mathbf{x}^{T} A \mathbf{x}$, then for any $\mathbf{x}, \mathbf{y} \in \mathbb{R}^{n}$, we have

$$
\sqrt{f(\mathbf{x}+\mathbf{y})} \leq \sqrt{f(\mathbf{x})}+\sqrt{f(\mathbf{y})} .
$$

Furthermore, if $A>0$, then the equality in (2) holds if and only if $\mathbf{x} \uparrow \mathbf{y}$.

Lemma 2.3 Let the function $\varphi:[0, c] \rightarrow(0, \infty)$ be defined by

$$
\varphi(u)=\sqrt{(c-u)^{2}+y^{2}-2(c-u) y \cos \theta}+u,
$$

where $c, u, y \in[0, \infty), \theta \in(0, \pi)$. Then the function $\varphi$ is nondecreasing. If in addition, $y>0$, then $\varphi$ is increasing.

Proof Note that

$$
\begin{aligned}
\frac{\partial \varphi}{\partial u} & =-\frac{(c-u)-\cos \theta y}{\sqrt{(c-u)^{2}+y^{2}-2(c-u) y \cos \theta}}+1 \\
& =\frac{\sqrt{(c-u)^{2}+y^{2}-2(c-u) y \cos \theta}-[(c-u)-\cos \theta y]}{\sqrt{(c-u)^{2}+y^{2}-2(c-u) y \cos \theta}} \\
& \geq \frac{\sqrt{(c-u)^{2}+y^{2}-2(c-u) y \cos \theta}-|(c-u)-\cos \theta y|}{\sqrt{(c-u)^{2}+y^{2}-2(c-u) y \cos \theta}} \\
& =\frac{\left[(c-u)^{2}+y^{2}-2(c-u) y \cos \theta\right]^{-1 / 2} \sin ^{2} \theta y^{2}}{\sqrt{(c-u)^{2}+y^{2}-2(c-u) y \cos \theta}+|(c-u)-\cos \theta y|} \\
& \geq 0 .
\end{aligned}
$$

Thus, $\varphi:[0, c] \rightarrow(0, \infty)$ is nondecreasing. Furthermore, if $y>0$, then $\varphi:[0, c] \rightarrow(0, \infty)$ is a strictly increasing function. This ends the proof.

Lemma 2.4 Let $B, C \in \mathbb{E}$. If $B \neq C$ and $D \in[B C]$, then

$$
\|C-B\|=\|C-D\|+\|D-B\| .
$$

The result of Lemma 2.4 is well known.

By our assumptions (H1.2)-(H1.5), we may easily get the following result.

Lemma 2.5 Let $\operatorname{CLS}\left\{\Gamma_{n}(\mathbf{A}), \Gamma_{N}\left(\mathbf{A}^{*}\right), \delta\right\}_{\mathbb{E}}$ be a CLS. Then, for any $i \in\{1,2, \ldots, n\}$, there exist $\sigma(i) \in\{1,2, \ldots, N\}$ and $\tau(i) \in\{0,1,2, \ldots, N-n\}$ such that

$$
A_{\sigma(i)+k}^{*} \in\left[A_{i} A_{i+1}\right), \quad k=0,1,2, \ldots, \tau(i) .
$$


Furthermore,

$$
\sum_{i=1}^{n} \tau(i)=N-n
$$

Lemma 2.6 Let $\operatorname{CLS}\left\{\Gamma_{n}(\mathbf{A}), \Gamma_{N}\left(\mathbf{A}^{*}\right), \delta\right\}_{\mathbb{E}}$ be a CLS. If the infimum of $\left|\Gamma_{N}\left(\mathbf{A}^{*}\right)\right|$ can be attained, then for any

$$
i \in\{1,2, \ldots, n\}, \quad k \in\{1,2, \ldots, \tau(i)\}, \quad \tau(i) \geq 1,
$$

we have

$$
\left\|A_{\sigma(i)+k}^{*}-A_{\sigma(i)+k-1}^{*}\right\|=\delta
$$

where $\sigma(i)$ and $\tau(i)$ are defined in Lemma 2.5 .

Proof Suppose to the contrary that there exist $i \in\{1,2, \ldots, n\}$ and $k \in\{1,2, \ldots, \tau(i)\}$ such that

$$
\left\|A_{\sigma(i)+k}^{*}-A_{\sigma(i)+k-1}^{*}\right\|>\delta .
$$

We construct a new $\operatorname{CLS}\left\{\Gamma_{n}(\mathbf{A}), \Gamma_{N}\left(\mathbf{A}^{* *}\right), \delta\right\}_{\mathbb{E}}$ as follows: If

$$
j \neq \sigma(i)+k, \quad k=0,1, \ldots, \tau(i)-1
$$

then $A_{j}^{* *}=A_{j}^{*}$. If there exists $k \in\{0,1, \ldots, \tau(i)-1\}$ such that $j=\sigma(i)+k$, then

$$
A_{j}^{* *} \in\left[A_{i} A_{\sigma(i)+\tau(i)}^{*}\right)
$$

and

$$
\left\|A_{\sigma(i)+1}^{* *}-A_{\sigma(i)}^{* *}\right\|=\left\|A_{\sigma(i)+2}^{* *}-A_{\sigma(i)+1}^{* *}\right\|=\cdots=\left\|A_{\sigma(i)+\tau(i)}^{* *}-A_{\sigma(i)+\tau(i)-1}^{* *}\right\|=\delta .
$$

Now fix $A_{\sigma(i)+\tau(i)}^{*} \in\left[A_{i} A_{i+1}\right)$. Denote

$$
\left(c_{i}, u_{i}, y_{i}\right):=\left(\left\|A_{\sigma(i)+\tau(i)}^{*}-A_{i}\right\|,\left\|A_{\sigma(i)+\tau(i)}^{*}-A_{\sigma(i)}^{*}\right\|,\left\|A_{\sigma(i-1)+\tau(i-1)}^{*}-A_{i}\right\|\right) .
$$

Without loss of generality, we can assume that $y_{i}>0, i=1,2, \ldots, n$. By condition (H1.3) and Lemma 2.4, we obtain that

$$
\left\|A_{\sigma(i)}^{*}-A_{i}\right\|=c_{i}-u_{i}, \quad c_{i} \geq u_{i}=\left\|A_{\sigma(i)+\tau(i)}^{*}-A_{\sigma(i)}^{*}\right\|=\sum_{k=1}^{\tau(i)}\left\|A_{\sigma(i)+k}^{*}-A_{\sigma(i)+k-1}^{*}\right\| .
$$

Since

$$
\|\boldsymbol{\alpha}-\boldsymbol{\beta}\|=\sqrt{\|\boldsymbol{\alpha}\|^{2}+\|\boldsymbol{\beta}\|^{2}-2\|\boldsymbol{\alpha}\| \cdot\|\boldsymbol{\beta}\| \cos \angle(\boldsymbol{\alpha}, \boldsymbol{\beta})}, \quad \forall \boldsymbol{\alpha}, \boldsymbol{\beta} \in \mathbb{E},
$$


we see that

$$
\begin{aligned}
\left|\Gamma_{N}\left(\mathbf{A}^{*}\right)\right| & =\sum_{j=1}^{n}\left(\left\|A_{\sigma(j)}^{*}-A_{\sigma(j-1)+\tau(j-1)}^{*}\right\|+\left\|A_{\sigma(j)+\tau(j)}^{*}-A_{\sigma(j)}^{*}\right\|\right) \\
& =\sum_{j=1}^{n}\left[\left\|\left(A_{\sigma(j)}^{*}-A_{i}\right)-\left(A_{\sigma(j-1)+\tau(j-1)}^{*}-A_{j}\right)\right\|+\left\|A_{\sigma(j)+\tau(j)}^{*}-A_{\sigma(j)}^{*}\right\|\right] \\
& =\sum_{j=1}^{n}\left[\sqrt{\left(c_{j}-u_{j}\right)^{2}+y_{j}^{2}-2\left(c_{j}-u_{j}\right) y_{j} \cos \angle A_{j}}+u_{j}\right] \\
& =\sum_{j=1}^{n} \varphi_{j}\left(u_{j}\right) \\
& =\sum_{1 \leq j \leq n, j \neq i} \varphi_{j}\left(u_{j}\right)+\varphi_{i}\left(u_{i}\right),
\end{aligned}
$$

where

$$
\varphi_{j}\left(u_{j}\right)=\sqrt{\left(c_{j}-u_{j}\right)^{2}+y_{j}^{2}-2\left(c_{j}-u_{j}\right) y_{j} \cos \angle A_{j}}+u_{j}, \quad j=1,2, \ldots, n .
$$

By condition (H1.6), (6) and (9), we see that

$$
u_{i}=\sum_{k=1}^{\tau(i)}\left\|A_{\sigma(i)+k}^{*}-A_{\sigma(i)+k-1}^{*}\right\|>\tau(i) \delta .
$$

According to Lemma 2.3, the function $\varphi_{i}:\left[0, c_{i}\right] \rightarrow(0, \infty)$ is increasing. Thus, by $(10)$ and (11), we have

$$
\left|\Gamma_{N}\left(\mathbf{A}^{*}\right)\right|=\sum_{1 \leq j \leq n, j \neq i} \varphi_{j}\left(u_{j}\right)+\varphi_{i}\left(u_{i}\right)>\sum_{1 \leq j \leq n, j \neq i} \varphi_{j}\left(u_{j}\right)+\varphi_{i}(\tau(i) \delta)=\left|\Gamma_{N}\left(\mathbf{A}^{* *}\right)\right| .
$$

This is contrary to the minimality of $\left|\Gamma_{N}\left(\mathbf{A}^{*}\right)\right|$. The proof is completed.

\section{Study of Problem 1.1}

\subsection{The case where $n$ is an odd number}

We first study the case of Problem 1.1 where $n$ is an odd number. In this situation we have the following result.

Theorem 3.1 Let $\operatorname{CLS}\left\{\Gamma_{n}(\mathbf{A}), \Gamma_{N}\left(\mathbf{A}^{*}\right), \delta\right\}_{\mathbb{E}}$ be a CLS and $n$ is an odd number. Then we have the following inequality:

$$
\left|\Gamma_{N}\left(\mathbf{A}^{*}\right)\right| \geq\left|\Gamma_{n}(\mathbf{A})\right| \sin \frac{\angle A}{2}+\left(1-\sin \frac{\angle A}{2}\right)(N-n) \delta .
$$

Proof We construct another $\operatorname{CLS}\left\{\Gamma_{n}(\mathbf{A}), \Gamma_{N}\left(\mathbf{A}^{* *}\right), \delta\right\}_{\mathbb{E}}$ such that (7) holds for any $i \in$ $\{1,2, \ldots, n\}$. Set

$$
\left\|A_{\sigma(j-1)+\tau(j-1)}^{* *}-A_{j}\right\|=y_{j}, \quad\left\|A_{\sigma(j)}^{* *}-A_{j}\right\|=x_{j} .
$$


By equality (7) and Lemma 2.4, we see that

$$
\begin{aligned}
y_{j+1} & =\left\|A_{j+1}-A_{\sigma(j)+\tau(j)}^{* *}\right\| \\
& =\left\|\left(A_{j+1}-A_{j}\right)-\left(A_{\sigma(j)+\tau(j)}^{* *}-A_{j}\right)\right\| \\
& =\left\|A_{j+1}-A_{j}\right\|-\left\|A_{\sigma(j)+\tau(j)}^{* *}-A_{j}\right\| \\
& =a_{j}-x_{j}-\tau(j) \delta,
\end{aligned}
$$

therefore,

$$
x_{j}+y_{j+1}=a_{j}-\tau(j) \delta, \quad j \in\{1,2, \ldots, n\} .
$$

Since

$$
\sum_{i=1}^{n} x_{i+1}=\sum_{i=1}^{n} x_{i}, \quad \sum_{i=1}^{n} y_{i+1}=\sum_{i=1}^{n} y_{i}
$$

by (13) and (14) we obtain that

$$
\sum_{j=1}^{n} x_{j}+\sum_{j=1}^{n} y_{j}=\sum_{j=1}^{n} a_{j}-\delta \sum_{j=1}^{n} \tau(j)=\left|\Gamma_{n}(\mathbf{A})\right|-\delta(N-n) .
$$

In view of Lemma 2.6 and equality (10), we see that

$$
\begin{aligned}
\left|\Gamma_{N}\left(\mathbf{A}^{*}\right)\right| & \geq\left|\Gamma_{N}\left(\mathbf{A}^{* *}\right)\right| \\
& =\sum_{j=1}^{n}\left(\left\|A_{\sigma(j)}^{* *}-A_{\sigma(j-1)+\tau(j-1)}^{* *}\right\|+\left\|A_{\sigma(j)+\tau(j)}^{* *}-A_{\sigma(j)}^{* *}\right\|\right) \\
& =\sum_{j=1}^{n}\left[\left\|\left(A_{\sigma(j)}^{* *}-A_{i}\right)-\left(A_{\sigma(j-1)+\tau(j-1)}^{* *}-A_{j}\right)\right\|+\left\|A_{\sigma(j)+\tau(j)}^{* *}-A_{\sigma(j)}^{* *}\right\|\right] \\
& =\sum_{j=1}^{n}\left[\sqrt{x_{j}^{2}+y_{j}^{2}-2 x_{j} y_{j} \cos \angle A_{j}}+\tau(j) \delta\right] \\
& \geq \sum_{j=1}^{n}\left[\sqrt{x_{j}^{2}+y_{j}^{2}-2 x_{j} y_{j} \cos \angle A}+\tau(j) \delta\right] \\
& =\sum_{j=1}^{n} \sqrt{x_{j}^{2}+y_{j}^{2}-2 x_{j} y_{j} \cos \angle A}+\sum_{j=1}^{n} \tau(j) \delta \\
& =\sum_{j=1}^{n} \sqrt{x_{j}^{2}+y_{j}^{2}-2 x_{j} y_{j} \cos \angle A}+\delta(N-n) \\
& =\sum_{j=1}^{n} \sqrt{f\left(x_{j}, y_{j}\right)^{T}+\delta(N-n),}
\end{aligned}
$$

i.e.,

$$
\left|\Gamma_{N}\left(\mathbf{A}^{*}\right)\right| \geq \sum_{j=1}^{n} \sqrt{f\left(x_{j}, y_{j}\right)^{T}}+\delta(N-n)
$$


where

$$
f\left(x_{j}, y_{j}\right)^{T}:=x_{j}^{2}+y_{j}^{2}-2 x_{j} y_{j} \cos \angle A=\left(x_{j}, y_{j}\right)\left[\begin{array}{cc}
1 & -\cos \angle A \\
-\cos \angle A & 1
\end{array}\right]\left(\begin{array}{l}
x_{j} \\
y_{j}
\end{array}\right) .
$$

Note that condition (H1.1) implies $0<\angle A<\pi$, thus, where the matrix is positive definite. According to inequality (15), Lemma 2.2 and equality (14), we obtain that

$$
\begin{aligned}
\left|\Gamma_{N}\left(\mathbf{A}^{*}\right)\right| & \geq \sum_{j=1}^{n} \sqrt{f\left(x_{j}, y_{j}\right)^{T}}+\delta(N-n) \\
& =\frac{1}{2}\left[\sum_{j=1}^{n} \sqrt{f\left(x_{j}, y_{j}\right)^{T}}+\sum_{j=1}^{n} \sqrt{f\left(x_{j}, y_{j}\right)^{T}}\right]+\delta(N-n) \\
& =\frac{1}{2}\left[\sum_{j=1}^{n} \sqrt{f\left(x_{j}, y_{j}\right)^{T}}+\sum_{j=1}^{n} \sqrt{f\left(y_{j}, x_{j}\right)^{T}}\right]+\delta(N-n) \\
& \geq \frac{1}{2} \sqrt{f\left\{\sum_{j=1}^{n}\left[\left(x_{j}, y_{j}\right)^{T}+\left(y_{j}, x_{j}\right)^{T}\right]\right\}}+\delta(N-n) \\
& =\frac{1}{2} \sqrt{f\left\{\left[\sum_{j=1}^{n}\left(x_{j}+y_{j}, y_{j}+x_{j}\right)^{T}\right]\right\}}+\delta(N-n) \\
& \left.\left.=\frac{1}{2} \sqrt{f\left\{\left[\sum_{j=1}^{n}\left(x_{j}+y_{j}\right), \sum_{j=1}^{n}\left(y_{j}+x_{j}\right)\right.\right.}\right]^{T}\right\}+\delta(N-n) \\
& =\frac{1}{2} \sqrt{f\left[\left(\sum_{j=1}^{n} x_{j}+\sum_{j=1}^{n} y_{j}\right)(1,1)^{T}\right]+\delta(N-n)} \\
& =\frac{1}{2}\left(\sum_{j=1}^{n} x_{j}+\sum_{j=1}^{n} y_{j}\right) \sqrt{f(1,1)^{T}+\delta(N-n)} \\
& \frac{1}{2}\left[\left|\Gamma_{n}(\mathbf{A})\right|-\delta(N-n)\right] \sqrt{2-2 \cos \angle A}+\delta(N-n) \\
& {[\mathbf{A}) \mid-\delta(N-n)] \sin \frac{\angle A}{2}+\delta(N-n) } \\
& \left.=\sin \frac{\angle A}{2}\right)(N-n) \delta . \\
&
\end{aligned}
$$

This means that inequality (12) holds.

In addition, from the above analysis we may easily see that the equality in (12) holds if (H3.1) $\Gamma_{n}(\mathbf{A})$ is an equiangular $n$-gon;

(H3.2) for any $i \in\{1,2, \ldots, n\}, \tau(i) \geq 1$, equality (5) holds;

(H3.3) equality (4) holds; and

(H3.4) there exist $x, y \in[0, \infty)^{n}$ such that

$$
\begin{cases}\left(x_{j}, y_{j}\right)^{T} \uparrow\left(x_{1}, y_{1}\right)^{T} \uparrow\left(y_{j}, x_{j}\right)^{T}, & j=1,2, \ldots, n, \\ x_{j}+y_{j+1}=a_{j}-\tau(j) \delta, & j=1,2, \ldots, n, \\ \sqrt{x_{j}^{2}+y_{j}^{2}-2 x_{j} y_{j} \cos \angle A} \geq \delta, & j=1,2, \ldots, n,\end{cases}
$$


where

$$
\left(x_{i}, y_{i}\right)^{T}=\left(x_{j}, y_{j}\right)^{T} \Leftrightarrow i \equiv j(\bmod n), \quad i, j=0, \pm 1, \pm 2, \ldots
$$

The proof is completed.

\subsection{The case where $\boldsymbol{n}$ is an even number}

Now, we consider the case of Problem 1.1 where $n$ is an even number.

Theorem 3.2 Let $\operatorname{CLS}\left\{\Gamma_{n}(\mathbf{A}), \Gamma_{N}\left(\mathbf{A}^{*}\right), \delta\right\}_{\mathbb{E}}$ be a CLS, $n$ is even, and let

$$
\sum_{i=1}^{n}(-1)^{j+1} a_{j} \geq 0
$$

Then we have the following two assertions:

(I) If

$$
\delta(N-n)>\sum_{j=1}^{n}(-1)^{j+1} a_{j}
$$

then we have

$$
\begin{aligned}
\left|\Gamma_{N}\left(\mathbf{A}^{*}\right)\right| \geq & \left\{\sin ^{2} \frac{\angle A}{2}\left[\left|\Gamma_{n}(\mathbf{A})\right|-\delta(N-n)\right]^{2}\right. \\
& \left.+4 \delta^{2} \cos ^{2} \frac{\angle A}{2} \min ^{2}\{\{\omega\}, 1-\{\omega\}\}\right\}^{1 / 2}+\delta(N-n),
\end{aligned}
$$

where

$$
\omega=\frac{\sum_{j=1}^{n}(-1)^{j+1} a_{j}+\delta(N-n)}{2 \delta}, \quad\{\omega\}=\omega-[\omega] \in[0,1),
$$

and $[\omega]$ is the Gaussian function.

(II) If

$$
\delta(N-n) \leq \sum_{j=1}^{n}(-1)^{j+1} a_{j}
$$

then we have

$$
\begin{aligned}
\left|\Gamma_{N}\left(\mathbf{A}^{*}\right)\right| \geq & \left\{\sin ^{2} \frac{\angle A}{2}\left[\left|\Gamma_{n}(\mathbf{A})\right|-\delta(N-n)\right]^{2}\right. \\
& \left.+\cos ^{2} \frac{\angle A}{2}\left[\sum_{j=1}^{n}(-1)^{j+1} a_{j}-\delta(N-n)\right]^{2}\right\}^{1 / 2}+\delta(N-n) .
\end{aligned}
$$

Proof First we consider the case where

$$
\delta(N-n)>\sum_{j=1}^{n}(-1)^{j+1} a_{j}
$$


We show that for any $i \in\{1,2, \ldots, n\}$, equality (7) holds by constructing a new $\operatorname{CLS}\left\{\Gamma_{n}(\mathrm{~A})\right.$, $\left.\Gamma_{N}\left(\mathbf{A}^{* *}\right), \delta\right\}_{\mathbb{E}}$. Since $n$ is an even number, by the proof of Theorem 3.1 and (13), we see that

$$
\sum_{j=1}^{n / 2}\left(x_{2 j-1}+y_{2 j}\right)=\sum_{j=1}^{n / 2} a_{2 j-1}-\delta \sum_{i=1}^{n / 2} \tau(2 j-1)
$$

and

$$
\begin{aligned}
\sum_{j=1}^{n / 2}\left(y_{2 j-1}+x_{2 j}\right) & =\left(y_{1}+x_{2}\right)+\left(y_{3}+x_{4}\right)+\cdots+\left(y_{n-1}+x_{n}\right) \\
& =\left(x_{2}+y_{3}\right)+\left(x_{4}+y_{5}\right)+\cdots+\left(x_{n-2}+y_{n-1}\right)+\left(x_{n}+y_{1}\right) \\
& =\sum_{j=1}^{n / 2}\left(x_{2 j}+y_{2 j+1}\right) \\
& =\sum_{j=1}^{n / 2} a_{2 j}-\delta \sum_{i=1}^{n / 2} \tau(2 j) .
\end{aligned}
$$

Inequality (15) is still valid where

$$
f\left(x_{j}, y_{j}\right)^{T}:=x_{j}^{2}+y_{j}^{2}-2 x_{j} y_{j} \cos \angle A=\left(x_{j}, y_{j}\right)\left[\begin{array}{cc}
1 & -\cos \angle A \\
-\cos \angle A & 1
\end{array}\right]\left(\begin{array}{l}
x_{j} \\
y_{j}
\end{array}\right)
$$

is a positive definite quadratic function. By means of inequality (15), Lemma 2.2, (18), (19) and (4), we then obtain that

$$
\begin{aligned}
& \left|\Gamma_{N}\left(\mathbf{A}^{*}\right)\right| \geq\left|\Gamma_{N}\left(\mathbf{A}^{* *}\right)\right| \\
& \geq \sum_{j=1}^{n} \sqrt{f\left(x_{j}, y_{j}\right)^{T}}+\delta(N-n) \\
& =\sum_{j=1}^{n / 2} \sqrt{f\left(x_{2 j-1}, y_{2 j-1}\right)^{T}}+\sum_{j=1}^{n / 2} \sqrt{f\left(x_{2 j}, y_{2 j}\right)^{T}}+\delta(N-n) \\
& =\sum_{j=1}^{n / 2} \sqrt{f\left(x_{2 j-1}, y_{2 j-1}\right)^{T}}+\sum_{j=1}^{n / 2} \sqrt{f\left(y_{2 j}, x_{2 j}\right)^{T}}+\delta(N-n) \\
& \geq \sqrt{f\left[\sum_{j=1}^{n / 2}\left(x_{2 j-1}, y_{2 j-1}\right)^{T}+\sum_{j=1}^{n / 2}\left(y_{2 j}, x_{2 j}\right)^{T}\right]}+\delta(N-n) \\
& =\sqrt{f\left[\sum_{j=1}^{n / 2}\left(x_{2 j-1}+y_{2 j}\right), \sum_{j=1}^{n / 2}\left(y_{2 j-1}+x_{2 j}\right)\right]^{T}}+\delta(N-n) \\
& =\sqrt{f\left[\sum_{j=1}^{n / 2} a_{2 j-1}-\delta \sum_{i=1}^{n / 2} \tau(2 j-1), \sum_{j=1}^{n / 2} a_{2 j}-\delta \sum_{i=1}^{n / 2} \tau(2 j)\right]^{T}}+\delta(N-n) \\
& =\sqrt{f\left[P_{n}-\delta \tau, Q_{n}-\delta(N-n-\tau)\right]^{T}}+\delta(N-n),
\end{aligned}
$$


i.e.,

$$
\left|\Gamma_{N}\left(\mathbf{A}^{*}\right)\right| \geq \sqrt{f\left[P_{n}-\delta \tau, Q_{n}-\delta(N-n-\tau)\right]^{T}}+\delta(N-n),
$$

where

$$
P_{n}:=\sum_{j=1}^{n / 2} a_{2 j-1} \in(0, \infty), \quad Q_{n}:=\sum_{j=1}^{n / 2} a_{2 j} \in(0, \infty), \quad P_{n} \geq Q_{n}
$$

and

$$
\tau:=\sum_{i=1}^{n / 2} \tau(2 j-1) \in\{0,1,2, \ldots, N-n\} .
$$

Note that

$$
\begin{aligned}
f & {\left[P_{n}-\delta \tau, Q_{n}-\delta(N-n-\tau)\right]^{T} } \\
& =\left(P_{n}-\delta \tau\right)^{2}+\left[Q_{n}-\delta(N-n-\tau)\right]^{2}-2\left(P_{n}-\delta \tau\right)\left[Q_{n}-\delta(N-n-\tau)\right] \cos \angle A \\
& =\left[P_{n}+Q_{n}-\delta(N-n)\right]^{2}-2(1+\cos \angle A)\left(P_{n}-\delta \tau\right)\left[Q_{n}-\delta(N-n-\tau)\right] \\
& =\left[P_{n}+Q_{n}-\delta(N-n)\right]^{2}-4 \cos ^{2} \frac{\angle A}{2}\left(P_{n}-\delta \tau\right)\left[Q_{n}-\delta(N-n-\tau)\right] \\
& =\sin ^{2} \frac{\angle A}{2}\left[\left|\Gamma_{n}(\mathbf{A})\right|-\delta(N-n)\right]^{2}+4 \delta^{2} \cos ^{2} \frac{\angle A}{2}\left[\frac{\sum_{j=1}^{n}(-1)^{j+1} a_{j}+\delta(N-n)}{2 \delta}-\tau\right]^{2} \\
& =\sin ^{2} \frac{\angle A}{2}\left[\left|\Gamma_{n}(\mathbf{A})\right|-\delta(N-n)\right]^{2}+4 \delta^{2} \cos ^{2} \frac{\angle A}{2}(\{\omega\}+[\omega]-\tau)^{2},
\end{aligned}
$$

i.e.,

$$
\begin{aligned}
f & {\left[P_{n}-\delta \tau, Q_{n}-\delta(N-n-\tau)\right]^{T} } \\
& =\sin ^{2} \frac{\angle A}{2}\left[\left|\Gamma_{n}\right|-\delta(N-n)\right]^{2}+4 \delta^{2} \cos ^{2} \frac{\angle A}{2}(\{\omega\}+[\omega]-\tau)^{2},
\end{aligned}
$$

where

$$
\omega=\frac{\sum_{j=1}^{n}(-1)^{j+1} a_{j}+\delta(N-n)}{2 \delta} .
$$

Set

$$
\tau_{0}= \begin{cases}{[\omega]} & \text { if } 0 \leq\{\omega\} \leq \frac{1}{2} \\ {[\omega]+1} & \text { if } \frac{1}{2}<\{\omega\}<1\end{cases}
$$

Since

$$
\delta(N-n)>\sum_{j=1}^{n}(-1)^{j+1} a_{j}, \quad \tau \in\{0,1, \ldots, N-n\},
$$


we see that

$$
\frac{\sum_{j=1}^{n}(-1)^{j+1} a_{j}+\delta(N-n)}{2 \delta}-(N-n) \leq\{\omega\}+[\omega]-\tau \leq\{\omega\}+[\omega] .
$$

Thus, if

$$
0 \leq\{\omega\} \leq \frac{1}{2}
$$

then

$$
\begin{aligned}
f & {\left[P_{n}-\delta \tau, Q_{n}-\delta(N-n-\tau)\right]^{T} } \\
& \geq \sin ^{2} \frac{\angle A}{2}\left[\left|\Gamma_{n}\right|-\delta(N-n)\right]^{2}+4 \delta^{2} \cos ^{2} \frac{\angle A}{2}\{\omega\}^{2},
\end{aligned}
$$

where equality holds if and only if $\tau=[\omega]=\tau_{0}$, and if

$$
\frac{1}{2}<\{\omega\}<1
$$

then

$$
\begin{aligned}
& f\left[P_{n}-\delta \tau, Q_{n}-\delta(N-n-\tau)\right]^{T} \\
& \quad \geq \sin ^{2} \frac{\angle A}{2}\left[\left|\Gamma_{n}(\mathbf{A})\right|-\delta(N-n)\right]^{2}+4 \delta^{2} \cos ^{2} \frac{\angle A}{2}(1-\{\omega\})^{2} .
\end{aligned}
$$

The equalities in (25) and (26) hold if and only if $\tau=[\omega]+1=\tau_{0}$. From (20), (21), (25) and (26), we see that

$$
\begin{aligned}
\left|\Gamma_{N}\left(\mathbf{A}^{*}\right)\right| \geq & \sqrt{f\left[P_{n}-\delta \tau, Q_{n}-\delta(N-n-\tau)\right]^{T}}+\delta(N-n) \\
\geq & \left\{\sin ^{2} \frac{\angle A}{2}\left[\left|\Gamma_{n}(\mathbf{A})\right|-\delta(N-n)\right]^{2}\right. \\
& \left.+4 \delta^{2} \cos ^{2} \frac{\angle A}{2} \min ^{2}\{\{\omega\}, 1-\{\omega\}\}\right\}^{1 / 2}+\delta(N-n) .
\end{aligned}
$$

Thus, inequality (16) is proved.

Second, we consider the case

$$
\delta(N-n) \leq \sum_{j=1}^{n}(-1)^{j+1} a_{j}
$$

Since

$$
\delta(N-n) \leq \sum_{j=1}^{n}(-1)^{j+1} a_{j}, \quad \tau \in\{0,1, \ldots, N-n\}
$$

we see that

$$
0 \leq \frac{\sum_{j=1}^{n}(-1)^{j+1} a_{j}+\delta(N-n)}{2 \delta}-(N-n) \leq\{\omega\}+[\omega]-\tau \leq\{\omega\}+[\omega]
$$


and

$$
\begin{aligned}
(\{\omega\}+[\omega]-\tau)^{2} & \geq\left[\frac{\sum_{j=1}^{n}(-1)^{j+1} a_{j}+\delta(N-n)}{2 \delta}-(N-n)\right]^{2} \\
& =\frac{1}{4 \delta^{2}}\left[\sum_{j=1}^{n}(-1)^{j+1} a_{j}-\delta(N-n)\right]^{2}
\end{aligned}
$$

where equality holds in (28) if and only if $\tau=N-n$. By (20), (21) and (28), we have

$$
\begin{aligned}
\left|\Gamma_{N}\left(\mathbf{A}^{*}\right)\right| \geq & \sqrt{f\left[P_{n}-\delta \tau, Q_{n}-\delta(N-n-\tau)\right]^{T}}+\delta(N-n) \\
\geq & \left\{\sin ^{2} \frac{\angle A}{2}\left[\left|\Gamma_{n}(\mathbf{A})\right|-\delta(N-n)\right]^{2}\right. \\
& \left.+\cos ^{2} \frac{\angle A}{2}\left[\sum_{j=1}^{n}(-1)^{j+1} a_{j}-\delta(N-n)\right]^{2}\right\}^{1 / 2}+\delta(N-n) .
\end{aligned}
$$

Thus, inequality (16) is proved.

Finally, the conditions for the equality in (16) to hold are as follows:

(H3.5) The $n$-gon $\Gamma_{n}(\mathbf{A})$ is an equiangular $n$-gon.

(H3.6) Equality (5) holds for any $i \in\{1,2, \ldots, n\}, \tau(i) \geq 1$.

(H3.7) Equality (4) holds.

(H3.8) $\sum_{i=1}^{n / 2} \tau(2 j-1)=\tau_{0} \in\{0,1,2, \ldots, N-n\}$, where $\tau_{0}$ is defined by (23).

(H3.9) There exist $x, y \in[0, \infty)^{n}$ such that

$$
\begin{cases}\left(x_{2 j-1}, y_{2 j-1}\right)^{T} \uparrow\left(x_{1}, y_{1}\right)^{T} \uparrow\left(y_{2 j}, x_{2 j}\right)^{T}, & j=1,2, \ldots, n / 2, \\ x_{j}+y_{j+1}=a_{j}-\tau(j) \delta, & j=1,2, \ldots, n, \\ \sqrt{x_{j}^{2}+y_{j}^{2}-2 x_{j} y_{j} \cos \angle A} \geq \delta, & j=1,2, \ldots, n .\end{cases}
$$

While the conditions for the equality in (17) to hold are as follows:

(H3.10) The conditions (H3.5)-(H3.7) and (H3.9) hold.

(H3.11) $\sum_{i=1}^{n / 2} \tau(2 j-1)=N-n$.

Here,

$$
\left(x_{i}, y_{i}\right)^{T}=\left(x_{j}, y_{j}\right)^{T} \Leftrightarrow i \equiv j(\bmod n), \quad i, j=0, \pm 1, \pm 2, \ldots
$$

This completes the proof of this theorem.

\subsection{The case $\Gamma_{n}(\mathrm{~A})$ is an equiangular $n$-gon}

Equiangular polygon is a special kind of polygons. Regular polygon in $\mathbb{R}^{2}$ is an equiangular polygon. If $\mathbb{E}$ is a Euclidean space with $\operatorname{dim} \mathbb{E} \geq 2$, then there is an equiangular 4-gon $\Gamma_{4}(\mathbf{A})$ in $\mathbb{E}$. Indeed, in $\mathbb{E}$, there exist at least two linearly independent vectors $\boldsymbol{\alpha}, \boldsymbol{\beta}$. Then, by the Gram-Schmidt orthogonalization process, we may obtain two orthogonal unit vectors $\mathbf{i}, \mathbf{j} \in \mathbb{E}$ from $\alpha, \beta$. If we set

$$
A_{1}=a\left(\mathbf{i} \cos \frac{\pi}{3}+\mathbf{j} \sin \frac{\pi}{3}\right), \quad A_{2}=-b\left(\mathbf{i} \cos \frac{\pi}{3}+\mathbf{j} \sin \frac{\pi}{3}\right), \quad A_{3}=-b \mathbf{i}, \quad A_{4}=a \mathbf{i},
$$


where $a, b \in(0, \infty)$, then the 4 -gon $\Gamma_{4}(\mathbf{A})$ is an equiangular polygon in $\mathbb{E}$ with

$$
\angle A_{1}=\angle A_{2}=\angle A_{3}=\angle A_{4}=\frac{\pi}{3}
$$

Similarly, if $\operatorname{dim} \mathbb{E} \geq 3$, then the 8 -gon

$$
\Gamma_{8}(\mathrm{~A}):=\Gamma_{8}(\mathbf{0}, \mathbf{i}, \mathbf{i}+\mathbf{j}, \mathbf{j}, \mathbf{j}+\mathbf{k}, \mathbf{i}+\mathbf{j}+\mathbf{k}, \mathbf{i}+\mathbf{k}, \mathbf{k})
$$

is equiangular with

$$
\angle A_{1}=\angle A_{2}=\cdots=\angle A_{8}=\frac{\pi}{2}
$$

where $\mathbf{i}, \mathbf{j}, \mathbf{k}$ are three mutually orthogonal unit vectors in $\mathbb{E}$.

We now turn to the calculation of $\inf \left\{\left|\Gamma_{N}\left(\mathbf{A}^{*}\right)\right|\right\}$.

Theorem 3.3 Let $\operatorname{CLS}\left\{\Gamma_{n}(\mathbf{A}), \Gamma_{N}\left(\mathbf{A}^{*}\right), \delta\right\}_{\mathbb{E}}$ be a CLS with $n$ odd, and let $\Gamma_{n}(\mathbf{A})$ be an equiangular n-gon. Suppose that there exist $\tau(i) \in\{0,1, \ldots, N-n\}$ for each $i=1,2, \ldots, n$, such that:

(H3.12) $\sum_{i=1}^{n} \tau(i)=N-n$.

(H3.13) $\frac{1}{2} \sum_{j=1}^{n}\left[(-1)^{j+1}\left(a_{j}-\tau(j) \delta\right)\right] \geq \frac{1}{2} \delta \csc \frac{\angle A}{2}$.

(H3.14) $(-1)^{k}\left\{\sum_{j=1}^{k-1}\left[(-1)^{j+1}\left(a_{j}-\tau(j) \delta\right)\right]-\frac{1}{2} \sum_{j=1}^{n}\left[(-1)^{j+1}\left(a_{j}-\tau(j) \delta\right)\right]\right\} \geq \frac{1}{2} \delta \csc \frac{\angle A}{2}$, where $k=2,3, \ldots, n$.

Then

$$
\inf \left\{\left|\Gamma_{N}\left(\mathbf{A}^{*}\right)\right|\right\}=\left|\Gamma_{n}(\mathbf{A})\right| \sin \frac{\angle A}{2}+\left(1-\sin \frac{\angle A}{2}\right)(N-n) \delta
$$

Proof By the assumptions in Theorem 3.3, conditions (H3.1) and (H3.3) hold. Since $\operatorname{dim} \mathbb{E} \geq 2$, there exists $\Gamma_{N}\left(\mathbf{A}^{*}\right) \subset \mathbb{E}$ such that condition (H3.2) holds. Thus, we just need to consider condition (H3.4).

From

$$
\left(x_{j}, y_{j}\right)^{T} \uparrow\left(x_{1}, y_{1}\right)^{T} \uparrow\left(y_{j}, x_{j}\right)^{T}, \quad j=1,2, \ldots, n,
$$

we have

$$
x_{j}=y_{j}, \quad j=1,2, \ldots, n \text {. }
$$

By

$$
x_{j}+y_{j+1}=a_{j}-\tau(j) \delta, \quad j=1,2, \ldots, n,
$$

and (30), we get

$$
y_{j}+y_{j+1}=a_{j}-\tau(j) \delta, \quad j=1,2, \ldots, n .
$$

We can rewrite (31) as

$$
(-1)^{j+1} y_{j+1}-(-1)^{j} y_{j}=(-1)^{j+1}\left(a_{j}-\tau(j) \delta\right), \quad j=1,2, \ldots, n .
$$


By (30), (32), $y_{n+1}=y_{1}$ and $n$ is odd number, we obtain that

$$
\begin{aligned}
& \sum_{j=1}^{k}\left[(-1)^{j+1} y_{j+1}-(-1)^{j} y_{j}\right]=\sum_{j=1}^{k}\left[(-1)^{j+1}\left(a_{j}-\tau(j) \delta\right)\right], \\
& (-1)^{k+1} y_{k+1}+y_{1}=\sum_{j=1}^{k}\left[(-1)^{j+1}\left(a_{j}-\tau(j) \delta\right)\right], \quad k=1,2, \ldots, n, \\
& x_{1}=y_{1}=\frac{1}{2} \sum_{j=1}^{n}\left[(-1)^{j+1}\left(a_{j}-\tau(j) \delta\right)\right], \\
& x_{k}=y_{k}=(-1)^{k}\left\{\sum_{j=1}^{k-1}\left[(-1)^{j+1}\left(a_{j}-\tau(j) \delta\right)\right]-\frac{1}{2} \sum_{j=1}^{n}\left[(-1)^{j+1}\left(a_{j}-\tau(j) \delta\right)\right]\right\},
\end{aligned}
$$

where $k=2,3, \ldots, n$. By $(30), x, y \in[0, \infty)^{n}$ and

$$
\sqrt{x_{j}^{2}+y_{j}^{2}-2 x_{j} y_{j} \cos \angle A} \geq \delta, \quad j=1,2, \ldots, n,
$$

we have

$$
x_{j}=y_{j} \geq \frac{1}{2} \delta \csc \frac{\angle A}{2}, \quad j=1,2, \ldots, n .
$$

According to the assumption in Theorem 3.3, and (33)-(35), condition (H3.4) holds. Consequently, by Theorem 3.1, Theorem 3.3 holds.

This completes the proof of Theorem 3.3.

Theorem 3.4 Let $\operatorname{CLS}\left\{\Gamma_{n}(\mathbf{A}), \Gamma_{N}\left(\mathbf{A}^{*}\right), \delta\right\}_{\mathbb{E}}$ be a CLS. Assume that $\Gamma_{n}(\mathbf{A})$ is an equiangular $n$-gon where $n$ is an even number, and

$$
\sum_{j=1}^{n}(-1)^{j+1} a_{j} \geq 0
$$

Suppose that there exist $\tau(i) \in\{0,1, \ldots, N-n\}$ for each $i=1,2, \ldots, n$ and free variable $y_{n} \in$ $(0, \infty)$ such that:

(H3.15) Condition (H3.12) holds.

(H3.16) The following inequalities hold:

$$
y_{j} \sqrt{\lambda^{2(-1)^{j+1}}+1-\lambda^{(-1)^{j+1}} \cos \angle A} \geq \delta, \quad j=1,2, \ldots, n,
$$

where

$$
\left(\begin{array}{c}
y_{1} \\
y_{2} \\
y_{3} \\
\vdots \\
y_{n-2} \\
y_{n-1}
\end{array}\right)=\left[\begin{array}{cccccc}
\lambda & 1 & 0 & 0 & 0 & 0 \\
\vdots & \ddots & \ddots & \vdots & \vdots & \vdots \\
0 & \cdots & \lambda^{(-1)^{j+1}} & 1 & \cdots & 0 \\
\vdots & \vdots & \vdots & \ddots & \ddots & \vdots \\
0 & 0 & 0 & 0 & \lambda^{-1} & 1 \\
0 & 0 & 0 & 0 & 0 & \lambda
\end{array}\right]^{-1}\left(\begin{array}{c}
a_{1}-\tau(1) \delta \\
\vdots \\
a_{j}-\tau(j) \delta \\
\vdots \\
a_{n-2}-\tau(j-2) \delta \\
a_{n-1}-\tau(n-1) \delta-y_{n}
\end{array}\right) \text {, }
$$




$$
\lambda=\frac{\sum_{j=1}^{n / 2}\left[a_{2 j-1}-\tau(2 j-1) \delta\right]}{\sum_{j=1}^{n / 2}\left[a_{2 j}-\tau(2 j) \delta\right]} .
$$

(H3.17)

$$
\sum_{j=1}^{n / 2} \tau(2 j-1)= \begin{cases}{[\omega],} & \delta(N-n)>\sum_{j=1}^{n}(-1)^{j+1} a_{j}, 0 \leq\{\omega\} \leq \frac{1}{2} \\ {[\omega]+1,} & \delta(N-n)>\sum_{j=1}^{n}(-1)^{j+1} a_{j}, \frac{1}{2}<\{\omega\}<1, \\ N-n, & \delta(N-n) \leq \sum_{j=1}^{n}(-1)^{j+1} a_{j}\end{cases}
$$

where $\omega$ is defined by (22).

Then we have the following two assertions:

(I) If

$$
\delta(N-n)>\sum_{j=1}^{n}(-1)^{j+1} a_{j}
$$

then

$$
\begin{aligned}
\inf \left\{\left|\Gamma_{N}\left(\mathbf{A}^{*}\right)\right|\right\}= & \left\{\sin ^{2} \frac{\angle A}{2}\left[\left|\Gamma_{n}(\mathbf{A})\right|-\delta(N-n)\right]^{2}\right. \\
& \left.+4 \delta^{2} \cos ^{2} \frac{\angle A}{2} \min ^{2}\{\{\omega\}, 1-\{\omega\}\}\right\}^{1 / 2}+\delta(N-n) .
\end{aligned}
$$

(II) If

$$
\delta(N-n) \leq \sum_{j=1}^{n}(-1)^{j+1} a_{j}
$$

then

$$
\begin{aligned}
\inf \left\{\left|\Gamma_{N}\left(\mathbf{A}^{*}\right)\right|\right\}= & \left\{\sin ^{2} \frac{\angle A}{2}\left[\left|\Gamma_{n}(\mathbf{A})\right|-\delta(N-n)\right]^{2}\right. \\
& \left.+\cos ^{2} \frac{\angle A}{2}\left[\sum_{j=1}^{n}(-1)^{j+1} a_{j}-\delta(N-n)\right]^{2}\right\}^{1 / 2}+\delta(N-n) .
\end{aligned}
$$

Proof We first look for the conditions for equality in (16)-(17) to hold. The conditions are either (H3.5)-(H3.9) or (H3.10)-(H3.11). By the assumptions in Theorem 3.4 and $\operatorname{dim} \mathbb{E} \geq$ 2, conditions (H3.5)-(H3.8) and (H3.11) hold. If (H3.5)-(H3.9) hold, then (H3.10) hold. Therefore we just need to show that (H3.9) holds.

Form (18)-(19) and

$$
\left(x_{2 j-1}, y_{2 j-1}\right)^{T} \uparrow\left(x_{1}, y_{1}\right)^{T} \uparrow\left(y_{2 j}, x_{2 j}\right)^{T}, \quad j=1,2, \ldots, \frac{n}{2},
$$

we see that

$$
\frac{x_{2 j-1}}{y_{2 j-1}}=\frac{y_{2 j}}{x_{2 j}}=\lambda, \quad j=1,2, \ldots, \frac{n}{2},
$$


where

$$
\lambda=\frac{\sum_{j=1}^{n / 2}\left(x_{2 j-1}+y_{2 j}\right)}{\sum_{j=1}^{n / 2}\left(y_{2 j-1}+x_{2 j}\right)}=\frac{\sum_{j=1}^{n / 2}\left[a_{2 j-1}-\tau(2 j-1) \delta\right]}{\sum_{j=1}^{n / 2}\left[a_{2 j}-\tau(2 j) \delta\right]} .
$$

Consequently,

$$
x_{j}=\lambda^{(-1)^{j+1}} y_{j}, \quad j=1,2, \ldots, n .
$$

By

$$
x_{j}+y_{j+1}=a_{j}-\tau(j) \delta, \quad j=1,2, \ldots, n,
$$

and (42), we have

$$
\lambda^{(-1)^{j+1}} y_{j}+y_{j+1}=a_{j}-\tau(j) \delta, \quad j=1,2, \ldots, n-1
$$

and

$$
\lambda^{-1} y_{n}+y_{1}=a_{n}-\tau(n) \delta .
$$

Equalities (40)-(43) imply that if $j=n$, then (43) holds, i.e., (44) holds. From (43) we have get (36), where $y_{n}$ are free variables. By $(42), x, y \in[0, \infty)^{n}$,

$$
\sqrt{x_{j}^{2}+y_{j}^{2}-2 x_{j} y_{j} \cos \angle A} \geq \delta, \quad j=1,2, \ldots, n,
$$

we have

$$
y_{j} \sqrt{\lambda^{2(-1)^{j+1}}+1-\lambda^{(-1)^{j+1}} \cos \angle A} \geq \delta, \quad j=1,2, \ldots, n .
$$

This means that condition (H3.9) can be deduced from conditions (H3.15)-(H3.17). Thus Theorem 3.4 holds by applying Theorem 3.2 .

This completes the proof of Theorem 3.4.

\section{Three effective examples}

For a general CLS $\left\{\Gamma_{n}(\mathbf{A}), \Gamma_{N}\left(\mathbf{A}^{*}\right), \delta\right\}_{\mathbb{E}}$, the equalities in (12), (16) and (17) may not hold, this is most probably because conditions (H3.1)-(H3.4) or conditions (H3.5)-(H3.11) cannot be met at the same time. We will discuss Problem 1.1 of a special CLS in $\mathbb{R}^{2}$.

Example 4.1 Consider the CLS $\left\{\triangle A B C, \Delta A^{*} B^{*} C^{*}, \delta\right\}_{\mathbb{R}^{2}}$, where

$$
A^{*} \in[B C), \quad B^{*} \in[C A), \quad C^{*} \in[A B), \quad 0<\delta<\min \{a, b, c\},
$$

and $a, b, c$ is the length of the sides of the triangle $\triangle A B C$. We will calculate that $\inf \left\{\left|\Delta A^{*} B^{*} C^{*}\right|\right\}$. 
By Theorem 3.1 we have

$$
\left|\Delta A^{*} B^{*} C^{*}\right| \geq|\triangle A B C| \sin \frac{\min \{A, B, C\}}{2},
$$

where $A, B, C$ are three inner angles of the triangle $\triangle A B C$. According to conditions (H3.1)(H3.4), the equality in (46) holds if and only if $\triangle A B C$ is a normal triangle, and $A^{*}, B^{*}$, $C^{*}$ are the midpoints of line segment $[B C],[C A],[A B]$, respectively. Consequently, the equality in inequality (12) does not hold in general.

It is well known that $\triangle A B C$ is an acute triangle if and only if

$$
\left[A A^{*}\right] \perp[B C], \quad\left[B B^{*}\right] \perp[C A], \quad\left[C C^{*}\right] \perp[A B]
$$

implies that $\left|\Delta A^{*} B^{*} C^{*}\right|$ takes the minimum. By this we see that

$$
\inf \left\{\left|\Delta A^{*} B^{*} C^{*}\right|\right\}=a \cos A+b \cos B+c \cos C \text {. }
$$

If $\pi / 2 \leq A<\pi$ and $\delta=0$, then

$$
B^{*}=C^{*}=A, \quad\left[A A^{*}\right] \perp[B C]
$$

is necessary and sufficient for $\left|\Delta A^{*} B^{*} C^{*}\right|$ to take the minimum. By this we see that

$$
\inf \left\{\left|\Delta A^{*} B^{*} C^{*}\right|\right\}=2\left|A A^{*}\right|=2 b \sin C=2 c \sin B .
$$

Example 4.2 Consider the $\operatorname{CLS}\left\{\Gamma_{3}(\mathbf{A}), \Gamma_{4}\left(\mathbf{A}^{*}\right), 1\right\}_{\mathbb{R}^{2}}$ (see Figure 2), where

$$
\left\|A_{1}-A_{2}\right\|=6, \quad\left\|A_{2}-A_{3}\right\|=8, \quad\left\|A_{3}-A_{2}\right\|=10
$$

and

$$
A_{1}^{*} \in\left[A_{1} A_{2}\right), \quad A_{2}^{*} \in\left[A_{2} A_{3}\right), \quad A_{3}^{*}, A_{4}^{*} \in\left[A_{3} A_{1}\right) .
$$

We will calculate that inf $\left\{\left|\Gamma_{4}\left(\mathbf{A}^{*}\right)\right|\right\}$.

Note that

$$
\cos \angle A_{1}=\frac{3}{5}, \quad \cos \angle A_{2}=0, \quad \cos \angle A_{3}=\frac{4}{5}
$$

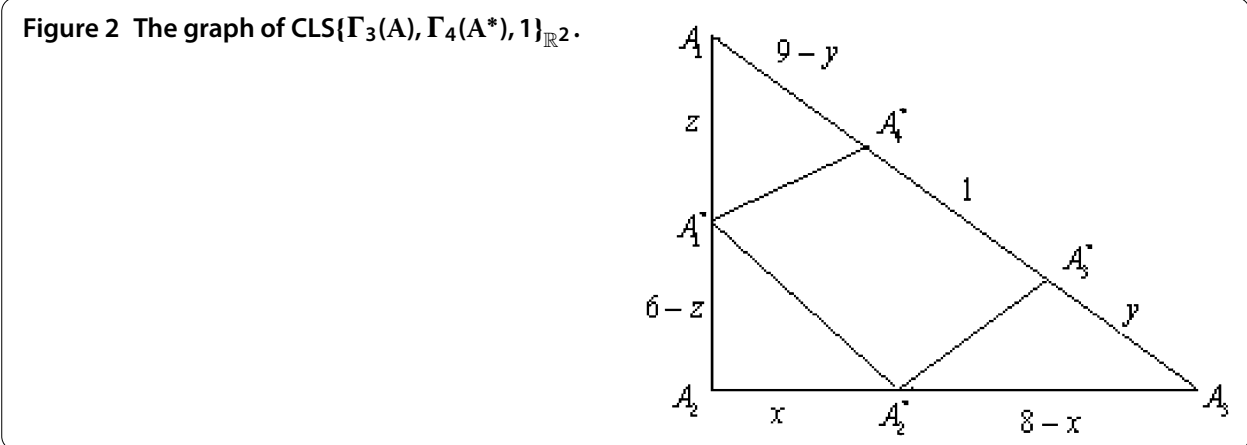


Without loss of generality, we may assume that $\left\|A_{4}^{*}-A_{3}^{*}\right\|=1$. By Lemma 2.6, we have

$$
\begin{aligned}
\left|\Gamma_{4}^{*}(\mathbf{A})\right| \geq & \psi(x, y, z) \\
= & \sqrt{x^{2}+(6-z)^{2}}+\sqrt{(8-x)^{2}+y^{2}-\frac{8}{5}(8-x) y} \\
& +\sqrt{(9-y)^{2}+z^{2}-\frac{6}{5}(9-y) z}+1
\end{aligned}
$$

where $(x, y, z) \in[0,6] \times[0,9] \times[0,6]$, and

$$
\left\{\begin{array}{l}
\sqrt{x^{2}+(6-z)^{2}} \geq 1 \\
\sqrt{(8-x)^{2}+y^{2}-\frac{8}{5}(8-x) y} \geq 1 \\
\sqrt{(9-y)^{2}+z^{2}-\frac{6}{5}(9-y) z} \geq 1
\end{array}\right.
$$

By the help of Mathematica software, we obtain that

$$
\begin{aligned}
\inf \left\{\left|\Gamma_{4}\left(\mathbf{A}^{*}\right)\right|\right\} & =\psi(0.621684 \ldots, 5.90012 \ldots, 5.1705 \ldots) \\
& =10.600001492661695 \ldots
\end{aligned}
$$

We note that (49) and

$$
\frac{\partial \psi(x, y, z)}{\partial x}=\frac{\partial \psi(x, y, z)}{\partial y}=\frac{\partial \psi(x, y, z)}{\partial z}=0
$$

hold if

$$
(x, y, z)=(0.621684 \ldots, 5.90012 \ldots, 5.1705 \ldots) .
$$

Example 4.3 Consider the $\operatorname{CLS}\left\{\Gamma_{4}(\mathbf{A}), \Gamma_{5}\left(\mathbf{A}^{*}\right), 2\right\}_{\mathbb{R}^{2}}$ (see Figure 1), where $\Gamma_{4}(\mathbf{A})$ is a rectangle, and

$$
\left\|A_{2}-A_{1}\right\|=\left\|A_{4}-A_{3}\right\|=6, \quad\left\|A_{3}-A_{2}\right\|=\left\|A_{1}-A_{4}\right\|=5
$$

and

$$
A_{1}^{*} \in\left[A_{1} A_{2}\right), \quad A_{2}^{*} \in\left[A_{2} A_{3}\right), \quad A_{3}^{*}, A_{4}^{*} \in\left[A_{3} A_{4}\right), \quad A_{5}^{*} \in\left[A_{4} A_{1}\right) .
$$

We will calculate that $\inf \left\{\left|\Gamma_{5}\left(\mathbf{A}^{*}\right)\right|\right\}$.

We may assume that $\left\|A_{4}^{*}-A_{3}^{*}\right\|=2$. By Lemma 2.6, we have

$$
\begin{aligned}
\left|\Gamma_{5}\left(\mathbf{A}^{*}\right)\right| \geq & \varphi(x, y, z, w) \\
= & \sqrt{x^{2}+(5-w)^{2}}+\sqrt{(6-x)^{2}+y^{2}} \\
& +\sqrt{(5-y)^{2}+z^{2}}+\sqrt{(4-z)^{2}+w^{2}}+2,
\end{aligned}
$$


where $(x, y, z, w) \in[0,6] \times[0,5] \times[0,4] \times[0,5]$, and

$$
\left\{\begin{array}{l}
\sqrt{x^{2}+(5-w)^{2}} \geq 2 \\
\sqrt{(6-x)^{2}+y^{2}} \geq 2 \\
\sqrt{(5-y)^{2}+z^{2}} \geq 2 \\
\sqrt{(4-z)^{2}+w^{2}} \geq 2
\end{array}\right.
$$

By the help of Mathematica software, we obtain that

$$
\begin{aligned}
\inf \{\varphi(x, y, z, w)\} & =\varphi(2.75296 \ldots, 3.24704 \ldots, 1.75296 \ldots, 2.24704 \ldots) \\
& =16.1421356237309 \ldots \\
& =10 \sqrt{2}+2 .
\end{aligned}
$$

On the other hand, by Theorem 3.2, we have

$$
\begin{aligned}
\left|\Gamma_{5}\left(\mathbf{A}^{*}\right)\right| & \geq\left|\Gamma_{n}(\mathbf{A})\right| \sin \frac{\angle A}{2}+\left(1-\sin \frac{\angle A}{2}\right)(N-n) \delta \\
& =10 \sqrt{2}+2 .
\end{aligned}
$$

It should be noted that the apparent error is caused by the computer. Therefore

$$
\inf \left\{\left|\Gamma_{5}\left(\mathbf{A}^{*}\right)\right|\right\}=10 \sqrt{2}+2
$$

We note that (51) holds if

$$
(x, y, z, w)=(2.75296 \ldots, 3.24704 \ldots, 1.75296 \ldots, 2.24704 \ldots)
$$

We can also give another intuitive proof of equation (52) as follows.

By Theorem 3.2, the equality in (51) holds if conditions (H3.5)-(H3.11) hold. In fact, there exist

$$
(x, y, z, w) \in[0,6] \times[0,5] \times[0,4] \times[0,5],
$$

such that (50) holds. From (H3.9) we get

$$
\frac{x}{5-w}=\frac{z}{5-y}=\frac{6-x}{y}=\frac{4-z}{w}=\frac{x+z+(6-x)+(4-z)}{(5-w)+(5-y)+y+w}=1,
$$

i.e.,

$$
\left\{\begin{array}{l}
x=5-w \\
y=1+w \\
z=4-w
\end{array}\right.
$$

Combined with (50), (53), we get

$$
\sqrt{2} \leq w \leq 4
$$


where $w$ is a free variable. This means that (51) holds if and only if (53)-(54) hold. This proves equation (52).

In addition, we can also prove (52) by Theorem 3.4 .

Example 4.3 is a geometry problem. However, we can see this example as a circuit layout problem of a family. In addition, this example also means that the equalities in (12), (16) and (17) can hold.

Remark 4.1 Since a Euclidean space is an abstract space, we will find the applications of CLS in theoretical fields such as statistics (see $[1,7]$ ), matrix theory (see [8]), geometry (see [6, 9-12]) and space science (see [1,9]), etc.

Remark 4.2 A large number of theories of algebra, analysis, geometry, computer (see [9, $12-14]$ ) with inequality are used in this paper, which can be found in the latest literature $[1,6-16]$.

\section{Competing interests}

The authors declare that they have no conflicts of interest to this work.

\section{Authors' contributions}

All authors contributed equally and significantly in this paper. All authors read and approved the final manuscript.

\section{Author details}

${ }^{1}$ College of Mathematics and Information Science, Chengdu University, Chengdu, Sichuan 610106, China. ${ }^{2}$ Department of Mathematics and Computer Science, Longyan University, Longyan, Fujian 364012, China.

\section{Acknowledgements}

This work was supported in part by the Natural Science Foundation of China (No. 61309015) and in part by the Foundation of Scientific Research Project of Fujian Province Education Department of China (No. JK2012049). The authors are deeply indebted to Professor Sui Sun Cheng, Tsing Hua University, Taiwan, for many useful comments and keen observations which led to the present improved version of the paper as it stands.

Endnote

a The angle between two nonzero vectors $B$ and $C$ is defined to be $\angle(B, C)=$ : $\arccos (\langle B, C\rangle /\|B\|\|C\|) \in[0, \pi]$.

Received: 12 September 2013 Accepted: 5 December 2013 Published: 30 Dec 2013

\section{References}

1. Wen, JJ, Han, TY, Cheng, SS: Inequalities involving Dresher variance mean. J. Inequal. Appl. 2013, 366 (2013). http://www.journalofinequalitiesandapplications.com/content/2013/1/366

2. Matejčka, L: Sharp bounds for the weighted geometric mean of the first Seiffert and logarithmic means in terms of weighted generalized Heronian mean. Abstr. Appl. Anal. 2013, Article ID 721539 (2013)

3. Gao, HY, Guo, JL, Yu, WG: Sharp bounds for power mean in terms of generalized Heronian mean. Abstr. Appl. Anal. 2011, Article ID 679201 (2011)

4. Chu, YM, Long, BY: Bounds of the Neuman-Sándor mean using power and identric means. Abstr. Appl. Anal. 2013, Article ID 832591 (2013)

5. Chu, YM, Hou, SW: Sharp bounds for Seiffert mean in terms of contraharmonic mean. Abstr. Appl. Anal. 2012, Article ID 425175 (2012)

6. Gardner, RJ: The Brunn-Minkowski inequality. Bull. Am. Math. Soc. 39, 355-405 (2002)

7. Wen, JJ, Zhang, ZH: Jensen type inequalities involving homogeneous polynomials. J. Inequal. Appl. 2010, Article ID 850215 (2010). doi:10.1155/2010/850215

8. Wen, JJ, Wang, WL: Chebyshev type inequalities involving permanents and their applications. Linear Algebra Appl. 422(1), 295-303 (2007)

9. Gao, CB, Wen, JJ: Theory of surround system and associated inequalities. Comput. Math. Appl. 63, 1621-1640 (2012)

10. Gao, CB, Wen, JJ: Several identities and inequalities involving Jordan closed curves. Appl. Math. E-Notes 8, 148-158 (2008)

11. Wen, JJ, Ke, R, Lu, T: A class of geometric inequalities involving k-Brocard distance. Chin. Q. J. Math. 21(2), 210-219 (2006)

12. Wen, JJ, Wang, WL: The inequalities involving generalized interpolation polynomial. Comput. Math. Appl. 56(4), 1045-1058 (2008)

13. Wen, JJ, Han, TY, Gao, CB: Convergence tests on constant Dirichlet series. Comput. Math. Appl. 62(9), $3472-3489$ (2011)

14. Wen, JJ, Cheng, SS: Closed balls for interpolating quasi-polynomials. Comput. Appl. Math. 30(3), 545-570 (2011) 
15. Pečarić, JE, Wen, JJ, Wang, WL, Tao, L: A generalization of Maclaurin's inequalities and its applications. Math. Inequal. Appl. 8(4), 583-598 (2005)

16. Wen, JJ, Wang, WL: The optimization for the inequalities of power means. J. Inequal. Appl. 2006, Article ID 46782 (2006). doi:10.1155/JIA/2006/46782

10.1186/1029-242X-2013-592

Cite this article as: Wen et al.: Sharp lower bounds involving circuit layout system. Journal of Inequalities and Applications 2013, 2013:592

Submit your manuscript to a SpringerOpen ${ }^{\circ}$ journal and benefit from:

- Convenient online submission

- Rigorous peer review

- Immediate publication on acceptance

Open access: articles freely available online

- High visibility within the field

- Retaining the copyright to your article

Submit your next manuscript at $>$ springeropen.com 